\title{
La maladie de Rendu-Osler : manifestations bucco-faciales et attitude thérapeutique en odontologie
}

\section{Rendu-Osler disease: orofacial manifestations and management in odontology}

\author{
BASSIMA CHAMI (1), AMINE CHERKAOUI (2), IHSSAN SAHNOUN (3), WAFAA EL WADY (1)
}

\begin{abstract}
RÉSUMÉ
La maladie de Rendu-Osler est une affection vasculaire héréditaire rare. A travers cet article, les auteurs rappellent les manifestations bucco-faciales de cette affection, qui lorsqu'elles sont associées à d'autres signes cliniques, sont suffisamment caractéristiques pour permettre à l'odontologiste d'évoquer le diagnostic. La prise en charge bucco-dentaire des patients atteints de cette affection requiert des précautions particulières parce qu'il existe deux risques importants : un risque hémorragique et un risque infectieux (Med Buccale Chir Buccale 2006; 12: 213-219).
\end{abstract}

mots clés : Télangiectasies, maladie de Rendu-Osler-Weber, télangiectasie hémorragique héréditaire

\section{SUMMARY}

The Rendu-Osler disease is a rare vascular hereditary affection. This article permits the identification of its orofacial manifestations which, when associated to other clinical signs, are characteristic enough to permit the odontologist to make the diagnosis. The orodental management of patients suffering from this disease requires particular precautions because of the existence of two major risks : a hemorrhagic and an infectious one. (Med Buccale Chir Buccale 2006; 12: 213-219).

key words : Telangiectasia, Rendu-Osler-Weber disease, hereditary hemorrhagic telangiectasia médecine buccale chirurgie buccale

VOL. $12, \mathrm{~N}^{\circ} 4$ 2006

page 213

1- Service d'Odontologie chirurgicale, Centre de consultation et de traitement dentaires, Faculté de Médecine dentaire,

6212 Rabat Instituts, Maroc

2- Service de Médecine interne, Hôpital Avicenne, 6212 Rabat, Maroc

3- Service de Parodontologie, Faculté de Médecine dentaire, 6212 Rabat Instituts, Maroc

Demande de tirés à part :

Bassima Chami 168 Cité El qods Alia Mohammedia Maroc e-mail : bassima.chami1@caramail.com 
médecine buccale chirurgie buccale

VOL. $12, \mathrm{~N}^{\circ} 4$ 2006

page 214
La maladie de Rendu-Osler-Weber ou télangiectasie hémorragique héréditaire est plus connue sous le nom de maladie Rendu-Osler. C'est une affection à transmission autosomique, dominante, caractérisée par de multiples malformations vasculaires cutanéo-muqueuses et viscérales, associées à un risque de saignements itératifs [13]. Elle est connue depuis longtemps, mais c'est Rendu qui a eu le mérite, en 1896, de la distinguer des autres affections hématologiques, en particulier de l'hémophilie. Osler, en 1901, a été le premier à donner une description complète de la maladie constituée par la triade caractéristique : épistaxis récidivantes, transmission héréditaire et télangiectasies multiples [10]. En 1907, Weber a décrit les différentes manifestations de la maladie et a insisté sur son caractère évolutif ; en 1909, Hanes a proposé le terme de télangiectasie hémorragique héréditaire ${ }^{[10]}$.

Sa prévalence est variable selon les zones géographiques. Aux USA, elle est de 10 à 20 cas/100000 habitants [11]. En France, Danemark et Japon, elle est estimée à 1 cas/8000 habitants ${ }^{[3]}$. Chez les personnes atteintes, la muqueuse buccale est souvent le siège de malformations vasculaires en rapport avec la maladie. Ces malformations, suffisamment caractéristiques pour permettre au praticien d'évoquer le diagnostic, sont présentées succinctement ainsi que les mesures adaptées à la prise en charge odontologique de ces malades afin d'éviter les risques relatifs à cette affectation.

\section{ETIOPATHOGÉNIE}

La maladie de Rendu-Osler est une maladie hétérogène sur le plan moléculaire, puisqu'elle est liée à de nombreuses mutations intéressant au moins deux gènes différents (ENG, pour endogline, est situé sur le locus 9q33-34 ; ACVR1, codant pour ALK1 - activin receptor- type II like kinase 1 -, sur le locus 12q 11-14 ) codant pour des récepteurs membranaires pour le TGBB ou transforming growth factor - $B$ (facteur de croissance impliqué dans la régulation de l'angiogénèse) ${ }^{[8,18]}$. Selon le gène impliqué, on distingue respectivement deux types de la maladie : la télangiectasie hémorragique héré- ditaire de type 1 et de type $2^{[1,8]}$. Elle présente aussi une grande variabilité d'expression clinique inter- et intrafamiliale [16].

Ses manifestations sont dues à des anomalies vasculaires qui se traduisent sur le plan histologique par un défaut de connexion entre les cellules endothéliales ${ }^{[14]}$. Ces dernières seraient incapables de produire les prolongements cytoplasmiques qui normalement se chevauchent avec ceux des cellules adjacentes. II en résulte une jonction insuffisante entre ces cellules et par conséquent une fragilité capillaire [14]. Ces capillaires, initialement d'apparence normale, prolifèrent et se dilatent sous l'effet de la pression sanguine, ce qui donne naissance à des télangiectasies ${ }^{[14]}$. En plus de ces altérations ultrastructurales, une dilatation localisée des veinules post-capillaires dont la paroi est dépourvue de fibres élastiques, peut être observée chez ces malades [23]. De nombreuses dilatations se développent et s'anastomosent parfois avec des artérioles elles-mêmes dilatées, donnant naissance à des fistules artério-veineuses [23]. L'ensemble de ces altérations vasculaires explique les éventuelles ruptures spontanées et les hémorragies survenant par des traumatismes mineurs.

\section{MANIFESTATIONS CLINIQUES}

La maladie de Rendu-Osler est une affection multisystémique, elle comporte des lésions vasculaires cutanéo-muqueuses et viscérales.

\section{Manifestations bucco-faciales Télangiectasies cutanéo-muqueuses}

Sur le revêtement cutanéo-muqueux, les malformations vasculaires se manifestent par des télangiectasies. Ces dernières sont responsables d'hémorragies pouvant réaliser la première, voire la seule manifestation clinique de la maladie ; elles constituent le deuxième motif de consultation initiale. Ces télangiectasies se présentent comme un réseau de capillaires dilatés ou comme un angiome stellaire, centré par un point rouge vif de la taille d'une grosse tête d'épingle (1 à $2 \mathrm{~mm}$ de diamètre) d'où irradie dans toutes les directions un réseau capillaire [12]. Certains auteurs les classent en trois 

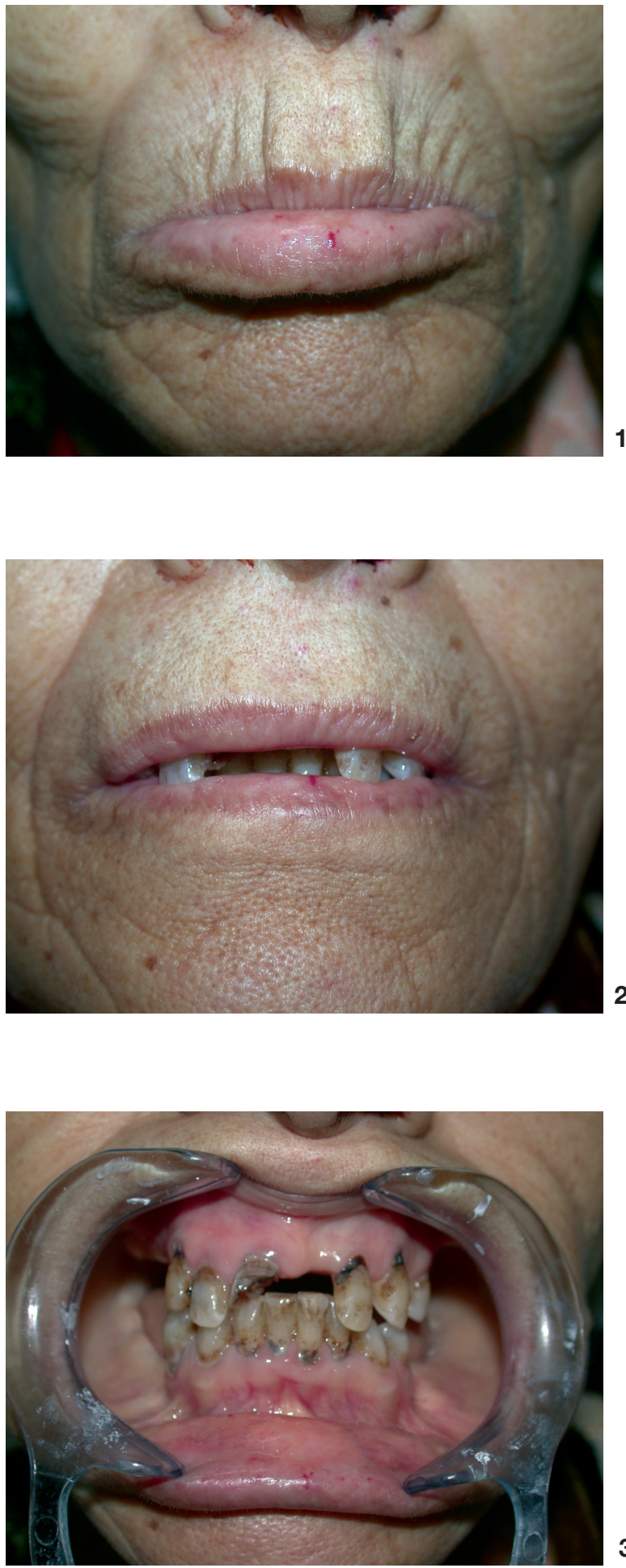

Figures 1, 2, 3 : Télangiectasies sur le bord libre et la face interne de la lèvre inférieure chez une patiente atteinte d'une maladie de Rendu-Osler.

Telangiectasias ont the free lingual margin and the internal surface of the lower lip in a patient suffering from the Rendu-Osler disease.

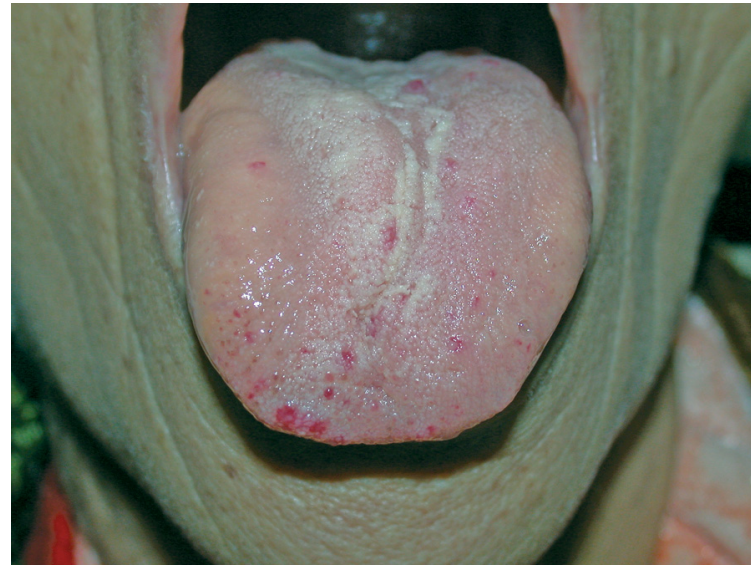

Figure 4 : Télangiectasies sur la face dorsale de la langue.

Telangiectasias on the dorsal surface of the tongue.

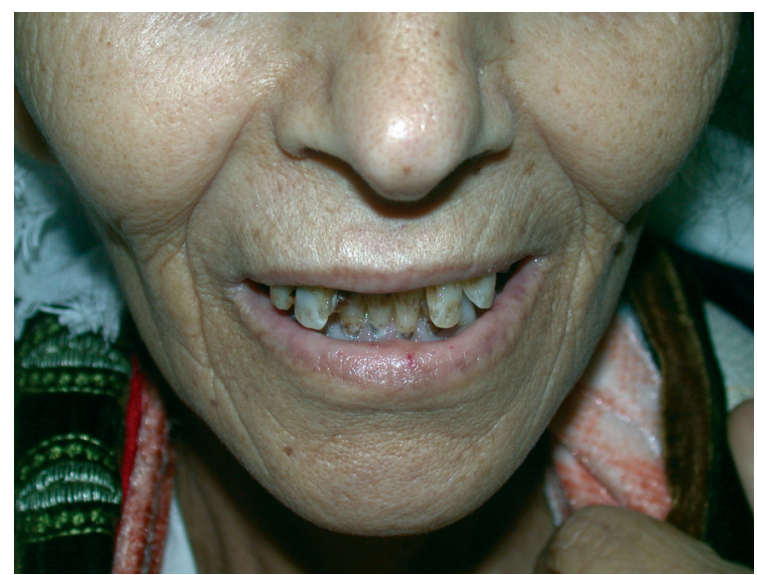

médecine

buccale

chirurgie

buccale

VOL. $12, \mathrm{~N}^{\circ} 4$

2006

page 215

Figure 5 : Télangiectasies sur le revêtement cutané facial.

Telangiectasias on the cutanous facial surface.

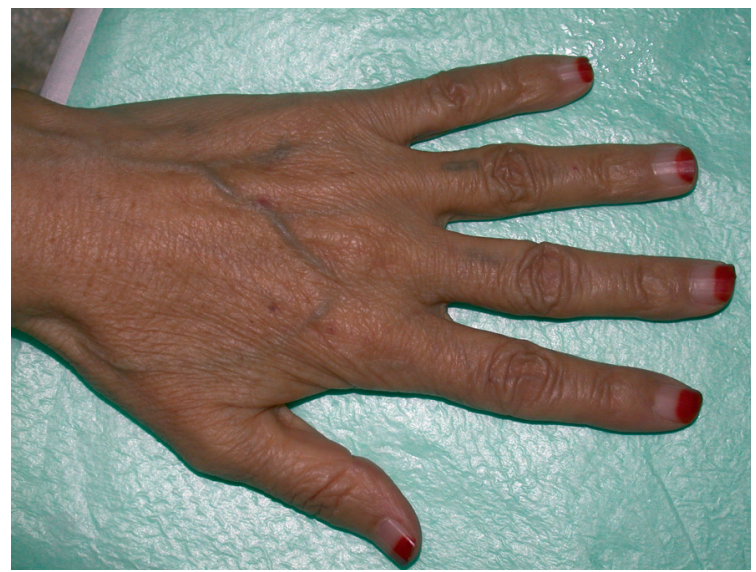

Figure 6 : Télangiectasies discrètes sur le revêtement cutané de la main gauche.

Discrete telangiectasias on the cutanous surface of the left hand. 
médecine

buccale

chirurgie

buccale

VOL. $12, \mathrm{~N}^{\circ} 4$ 2006

page 216 variétés morphologiques : lésions dont la taille est inférieure à $1 \mathrm{~mm}$, lésions nodulaires de plus grande taille et angiomes stellaires [12] sur la muqueuse buccale. Les lésions siègent le plus souvent sur la demi-muqueuse labiale [25], mais elles peuvent s'observer sur la face dorsale de la langue, la face interne des joues, les gencives, la muqueuse vélo-palatine et la paroi pharyngée (Fig. 1, 2, 3 et 4). Les télangiectasies gingivales pourraient être associées, pour des raisons inconnues, à la survenue des résorptions radiculaires cervicales sur les dents situées à proximité de ces lésions ${ }^{[7]}$. Sur le revêtement cutané, les télangiectasies prédominent sur le visage mais elles intéressent parfois les extrémités. Elles se développent en général après 40 ans (Fig. 5 et 6).

\section{Epistaxis}

Les épistaxis représentent la manifestation la plus fréquente de la maladie de Rendu-Osler : elles touchent 80 à $90 \%$ des patients [23]. Elles constituent volontiers la manifestation inaugurale et sont particulières par leur survenue spontanée, récurrente, nocturne, sans aucun facteur favorisant [15] (Fig.7). Elles sont dues à des dilatations angiomateuses des vaisseaux du septum, du plancher et des parois externes des fosses nasales [16]. Elles apparaissent souvent dans la deuxième décennie de la vie. Elles sont responsables, chez la plupart des patients de plus de 45 ans, de l'apparition d'une anémie ferriprive ${ }^{[19] .}$

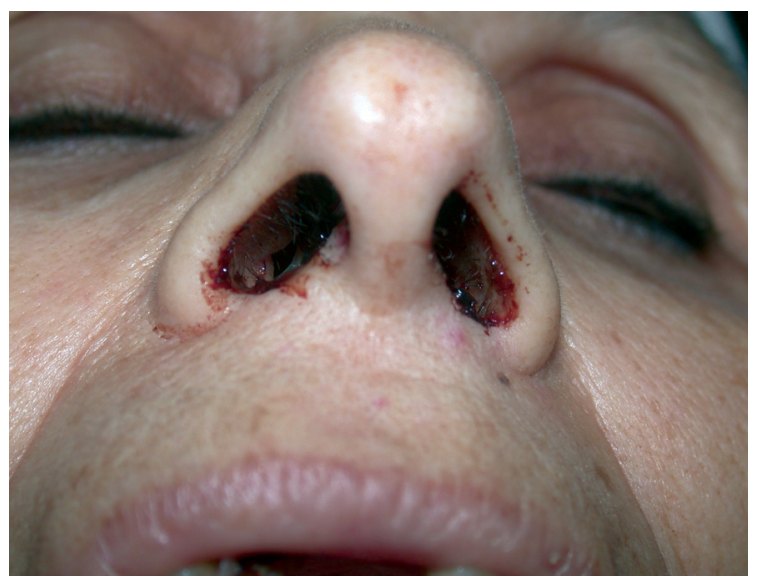

Figure 7 : Epistaxis bilatérale spontanée. Spontaneous bilateral epistaxis.

\section{Manifestations viscérales}

Les atteintes viscérales sont multiples. Elles intéressent plusieurs organes: d'abord les poumons, le système nerveux, le tube digestif...

\section{Atteintes pulmonaires}

Elles sont plus fréquentes chez les patients ayant le type 1 de la maladie ${ }^{[1,8]}$. Elles se présentent sous forme de fistules artério-veineuses constituées par des communications artério-veineuses dilatées, sans interposition d'un réseau capillaire. Ces malformations vasculaires se développent surtout dans les lobes inférieurs des poumons (65\%) [9, 13, 24] et elles sont présentes chez 5 à $15 \%$ des patients atteints de la maladie de Rendu-Osler [13, 24]. Leur taille augmente avec l'âge et au cours de la grossesse. Elles sont responsables d'une inactivation localisée de l'effet filtre du lit capillaire pulmonaire ainsi que d'une diminution de la capacité d'oxygénation du sang dans le territoire touché qui peut se traduire par une hypoxie et une polyglobulie compensatoire [13]. Elles sont le plus souvent asymptomatiques, se révélant parfois par des hémoptysies, une dyspnée d'effort ou une cyanose [6, 9]. Cependant, elles peuvent être responsables d'embolies paradoxales vers la circulation cérébrale [13].

\section{Complications neurologiques}

Elles émaillent l'évolution de la maladie de RenduOsler dans 10 à $30 \%$ des cas. Dans un tiers des cas, elles sont l'expression de malformations artério-veineuses spinales et/ou cérébrales [13]. Les localisations intracrâniennes sont souvent paucisymptomatiques (céphalées), mais parfois responsables de manifestations plus graves (épilepsie, hémorragie cérébrale, accident ischémique sur thrombose ou embolie) [13]. Dans les deux autres tiers des cas, les complications neurologiques sont secondaires aux malformations artério-veineuses pulmonaires $[4,13]$. Ces dernières sont à l'origine d'emboles, septiques ou non, responsables d'abcès cérébraux ou d'accidents ischémiques souvent révélateurs des malformations pulmonaires, voire de la maladie de Rendu-Osler elle-même [13]. Trois mécanismes physiopathologiques ont été proposés pour expliquer les accidents vasculaires cérébraux ischémiques : 
- Embolie paradoxale à partir d'un foyer de thrombophlébite périphérique - l'embole empruntant la fistule pulmonaire - ou éventuellement formation d'un thrombus au sein de la malformation artério-veineuse elle-même ;

- Embolie gazeuse par mise en communication d'un vaisseau pulmonaire avec les voies aériennes ;

- Polyglobulie responsable d'une thrombose artérielle in situ.

\section{Complications digestives}

L'hémorragie digestive est la complication la plus à craindre puisque plus de $40 \%$ des malades ont des épisodes hémorragiques gastro-intestinaux récurrents en rapport avec des télangiectasies de la muqueuse digestive. Ces hémorragies sont souvent tardives et touchent des malades ayant 50 ans et plus [6, 10].

\section{Atteintes hépatiques}

Elles surviennent chez 8 à $31 \%$ des patients [22, 24]. Les télangiectasies et les anastomoses artério-veineuses caractéristiques de la maladie constituent là aussi les lésions dominantes [1]. Ces atteintes paraissent mineures en particulier par rapport aux autres lésions cutanéo-muqueuses et digestives. Les conséquences physiopathologiques de ces malformations vasculaires sont essentiellement hémodyna- miques [22]. Les fistules artério-veineuses sus-hépatiques peuvent conduire à une décompensation cardiaque par augmentation du débit, alors que les fistules hépato-portales sont responsables de l'apparition d'une hypertension portale ${ }^{[5,22]}$ qui peut être compliquée par des varices œsophagiennes, une ascite ou une encéphalopathie [24]. D'autres complications, sont parfois observées : ischémie biliaire, lithiase intra-hépatique ou cirrhose biliaire ${ }^{[24]}$ mais elles restent assez rares.

\section{Autres manifestations}

Elles sont exceptionnelles. II s'agit d'hémorragies intéressant les autres muqueuses. Les manifestations oculaires sont rares (angiome rétinien, larmes de sang dues à la rupture de télangiectasies conjonctivales) [19].

\section{DIAGNOSTIC}

Le diagnostic de la maladie de Rendu-Osler est essentiellement clinique. II se base sur des critères établis lors de la conférence internationale de consensus qui s'est tenue à Curaçao en 2000 (Tab. 1) [3, 8, 16] . Quatre critères ont été retenus pour établir le diagnostic. La pénétrance des signes est liée à l'âge : elle devient complète vers l'âge de 40 ans [16]. Le diagnostic différentiel ne se discute que chez les médecine buccale chirurgie buccale

VOL. $12, N^{\circ} 4$ 2006

page 217

Tableau 1 : Critères pour le diagnostic de la maladie de Rendu-Osler (Conférence internationale de Consensus de Curaçao, 2000)

Criteria for the diagnostic of the Rendu-Osler disease (International Conference of Consensus from Curaçao, 2000)

\section{Critères diagnostiques}

1. Epistaxis

2. Télangiectasies

3. Atteintes viscérales

4. Histoire familiale

Diagnostic

Certain

3 critères présents

Possible ou suspecté

2 critères présents

Peu probable
Spontanées et récurrentes ; leur survenue nocturne est évocatrice

Multiples, siégeant dans des sites électifs : lèvres, cavité buccale, doigts, nez

Malformations artério-veineuses pulmonaires

Télangiectasies gastro-intestinales avec ou sans complications hémorragiques

Malformations artério-veineuses hépatiques

Malformations artério-veineuses cérébrales

Malformations artério-veineuses médullaires

Notion de parent du premier degré atteint de la maladie selon ces mêmes critères 
malades ne présentant pas forcément tous les signes de la maladie et surtout chez ceux qui présentent un seul des signes évocateurs [17]. Ainsi :

- Devant des télangiectasies cutanées siégeant principalement sur les extrémités des membres : il faut chercher les autres signes du CREST syndrome, à savoir des calcifications, un syndrome de Raynaud et des signes de sclérodermie [17].

- L'association d'épistaxis à répétition et de télangiectasies peu typiques dans leurs localisations et leur aspect peut être rencontrée lors d'une cirrhose hépatique. L'absence de caractère familial et la présence du facteur toxique sont des éléments précieux à prendre en compte [19] ;

- Les télangiectasies isolées doivent être différenciées des taches rubis très fréquemment observées sur le thorax, l'abdomen et le dos des sujets âgés. Ces dernières qui ne sont jamais en relief et qui sont parfois violacées, ne disparaissent pas médecine buccale chirurgie buccale

VOL. $12, \mathrm{~N}^{\circ} 4$ 2006

page 218 à la vitropression [17].

- L'épistaxis isolée doit faire évoquer une hypertension artérielle, une hémophilie, une thrombopénie... ou faire rechercher une étiologie nasale [17].

\section{PRISE EN CHARGE}

\section{Prise en charge générale}

Elle est pluridisciplinaire et dépend du siège des malformations vasculaires prédominantes et du retentissement sur l'état général du patient. Ainsi, les épistaxis doivent bénéficiées d'une prise en charge adéquate en $\mathrm{ORL}$ en raison de leur caractère récurrent et de l'anémie qu'elles peuvent engendrer à long terme [19]. II en est de même pour les autres manifestations dont certaines mettent parfois en jeu le pronostic vital du patient.

\section{Prise en charge bucco-dentaire}

Les patients atteints de la maladie de Rendu-Osler présentent essentiellement deux risques en odontologie : risque d'hémorragie et risque d'abcès cérébral secondaire à la bactériémie.

Le risque hémorragique est lié à la fragilité capillaire et aux traumatismes qui peuvent léser les télangiectasies siégeant sur la muqueuse buccale. Afin de diminuer ce risque, plusieurs mesures préventives et curatives peuvent être proposées :
- Mesures préventives : la prédisposition au saignement, spontané ou provoqué, impose une hygiène bucco-dentaire rigoureuse ainsi que des visites régulières chez l'odontologiste afin d'éviter toute évolution nécessitant des extractions dentaires ou tout autre acte chirurgical pouvant être responsable d'un saignement parfois difficile à juguler.

- Mesures curatives : devant une hémorragie buccale ou lors d'un geste chirurgical, il faut disposer des moyens techniques pour réaliser une hémostase locale (moyens de compression, électrocoagulation, agents hémostatiques...). Les lésions angiomateuses peuvent être excisées chirurgicalement, ou traitées par électrocoagulation ou cryothérapie ${ }^{12,21]}$. Le laser a été également conseillé, en particulier pour le traitement des télangiectasies gingivales [11, 21]. II produit une rétraction thermique qui provoque l'occlusion partielle des petits vaisseaux ; ils seront oblitérés secondairement par une thrombose.

Le risque de survenue d'abcès cérébral est beaucoup plus important chez les sujets atteints d'une maladie de Rendu-Osler. L'abcès cérébral est du à un embole septique qui passe à travers une fistule artério-veineuse pulmonaire. Ces emboles septiques peuvent avoir plusieurs origines : ostéomyélites, sinusites, abcès... [15]. Les foyers infectieux buccodentaires et surtout les bactériémies provoquées par les soins bucco-dentaires peuvent également

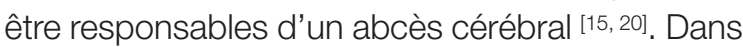
ce cas, les germes retrouvés dans l'abcès cérébral appartiennent à la flore bactérienne buccale [15]. Ce risque lié à la bactériémie impose chez ces patients une éradication de tous les foyers latents et surtout la prescription d'une antibioprophylaxie lors des soins bucco-dentaires invasifs. Le protocole d'antibioprophylaxie est similaire à celui proposé pour la prophylaxie de l'endocardite d'Osler [2, 3, 7, 11, 15].

\section{CONCLUSION}

La maladie de Rendu-Osler est une maladie héréditaire autosomique dominante, caractérisée par des lésions angiodysplasiques intéressant la muqueuse, le revêtement cutané et certains viscères. La région bucco-faciale constitue un siège 
fréquent des manifestations de cette maladie. L'odontologiste doit être capable de les dépister et de les relier à cette affection. II doit aussi connaître les différents risques auxquels ces patients sont exposés et prendre les précautions nécessaires pour la réalisation de soins buccodentaires.

\section{RÉFÉRENCES}

1 - Abdalla Sa, Geisthoff UW, Bonneau D, Plauchu H, McDonald J, Kennedy S, Faughnan ME, Letarte M. Viceral manifestations in hereditary haemorrhagic telangiectasia type 2. J Med Genet 2003 ; 40 : 494-502.

2 - AFSSAPS. Prescription des antibiotiques en odontologie et stomatologie, méthodologie et recommandations. Act Odont Stomatol 2002 ; 21 : 353-8.

3 - Begbie ME, Wallace GM, Shovlin CL. Hereditary haemorrhagic telangiectasia (Osler-Weber-Rendu syndrome): a review from the $21 \mathrm{St}$ Century. Postgrad Med J $2003 ; 79: 18-24$.

4- Broudet S, houdart E, Boissonnet H, Lot G. Hémorragies cérébroméningées de la maladie de Rendu-Osler : des cas traités par embolisation. Presse Med 1997 ; 26 : 1622-4.

5 - Buscarini E, Danesino C, Olivieri C, Lupinacci G, ZAMBELLI A. Liver involvement in hereditary haemorrhagic telangiectasia or Rendu-Osler-Weber disease. Dig Liver Dis 2005 ; 35 : 635-45.

6 - DONG SL, REYNolds SF, SteInER IP. Brain abscess in patients with hereditary hemorrhagic telangiectasia: case report and literature review. J Emerg Med 2001 ; $20: 247-51$

7 - EDWARDS PC, Mc VANEY T. External cervical root resorption involving multiple maxillary teeth in patient with hereditary hemorrhagic telangiectasia. Oral Surg Oral Med Oral Oral Path Oral Radiol Endod 2005 ; 100 : 585-91.

8 - FuchizaKI U, MiYamoI H, Kitagawa S, KaneKo S, KOBAYASHI K. Hereditary haemorrhagic telangiectasia (Rendu-Osler-Weber disease). Lancet 2003 ; 362 : 1490-4.

9 - Halefoglu AM. Rendu-Osler-Weber syndrome presenting with pulmonary arterioveinous fistula. Aust Radiol $2005 ; 49: 242-5$.

10 - Kitamura T, Tanabe S, Kolzumi W, Ohida M, Saigengl K, Мгтом H. Rendu-Osler-Weber disease successfully treated by argon plasma coagulation. Gastrointest Endos $2001 ; 54: 525-7$.

11 - LARRY FINKBEINER R. Progressive laser treatment of hereditary hemorrhagic telangiectasia of the gingival: case report. Int J Periodontics Resorative Dent 2004 ; 24 : 288-94.

12 - LASKARIS G. Maladies génétiques. Atlas des maladies buccales, Flammarion, Paris, 1994.
13 - LeCLeRCQ P, ThOmas A, SADZOt B. La maladie de RenduOsler : une cause rare d'AVC ischémique chez un sujet jeune. Rev Med (Liège) 2005 ; 60 : 641-6.

14 - MARX RE, SteRn D. Conditions of developmental disturbances. Oral and maxillofacial pathology: a rationale for diagnosis and treatment. Quintessence publishning Co, Chicago, 2003.

15 - Mohler ER, Monaham B, Don Canty M, Flockhart D. Cerebral abscess associated with dental procedure in hereditary haemorrhagic telangiectasia. Lancet 1991; $338: 508-9$.

16 - PERDU J. Maladies artérielles mendéliennes. Arch Cœur Vaisseaux 2003 ; 96 :1096-104.

17 - Plauchu H. Maladie de Rendu-Osler : quelle surveillance? Rev Prat 2004 ; 18 : 333-6.

18 - Plauchu H, Giraud S, Babin E, Sobrier F. Conseil génétique dans la maladie de Rendu- Osler. Rev Prat 2004 ; 18:380-1.

19 - Plauchu H, Brunet G, Bideau A, Robert. JM. La maladie de Rendu-Osler. Concours Med 1992 ; 114 : 2469 76.

20 - Russi EW, Dazzi H, GaumanN N. Septic pulmonary embolism due to periodontal disease in patient with hereditary hemorrhagic telangiectasia. Respiration 1996 ; 63 : 117-9.

21 - SAPPE JP, EVERSOLE LR, WYSOCKI GP. Bleeding disorders. Contemporary oral and maxillofacial pathology. Mosby, Saint Louis, 2004.

22 - Saurin JC, Dumortier J, Menar Y, Henry L, Bolllot O, PlaUChu H, Pliard P. Les malformations vasculaires hépatiques de la maladie de Rendu-Osler. Gastroenterol Clin Biol 2000 ; 24 : 89-93.

23 - Serrano E, Percodani J, Pessey JJ. La fermeture bilatérale des fosses nasales : une technique chirurgicale originale du traitement des épistaxis récidivantes graves de la maladie de Rendu-Osler. Ann Otolaryngol Chir Cervicofac 1998 ; 115 : 169-73.

24 - SUKKARIEH F, BRASSEUR P. Malformations artérioveineuses pulmonaires et hépatiques dans un cas de maladie de Rendu-Osler. J Radiol 2003 ; 84 ; 405-8.

25 - Wood NK, GoAz PW. Lesions of the lips. Differential diagnosis of oral and maxillofacial lesions. Mosby, Saint Louis, 1997.

\begin{tabular}{l}
$\begin{array}{l}\text { médecine } \\
\text { buccale } \\
\text { chirurgie } \\
\text { buccale }\end{array}$ \\
\hline VOL. $12, N^{\circ} 4$ \\
2006 \\
\hline page 219
\end{tabular}

page 219 


\section{COURS DE CHIRURGIE PRE-IMPLANTAIRE " De la greffe osseuse à la prothèse "}

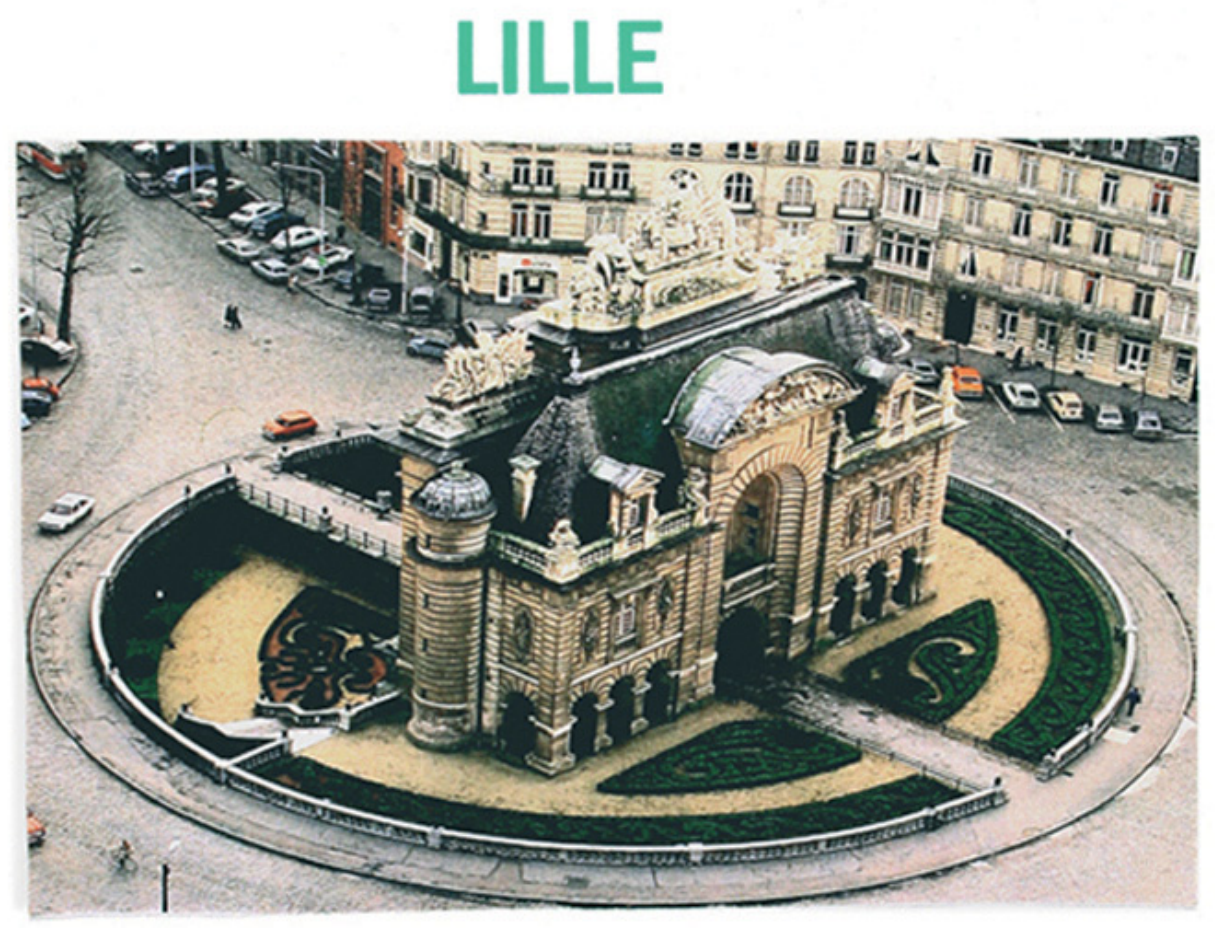

Vendredi 9 et Samedi 10 Mars 2007

\section{Organisé par: Le Professeur Joël FERRI}

\section{Renseignements et Inscription}

MFRD 2 Avenue de la créativité

59650 Villeneuve d'Ascq

Tél : 0320918950

Email : mfrd@neuf.fr
Secrétariat scientifique

Mme MANDERICK Service de Stomatologie

et Chinurgie Maxillo-Faciale CHRU Lille

Tél: 0320446376

Email：n-manderick@chru-lille.fr 